\title{
Sitzung vom 28. Februar 1876.
}

Vorsitzender: Hr. A. W. Hofmann, Präsident.

Das Protokoll der letzten Sitzung wird nach folgendem Zwischenfall genehmigt.

Der in der letzten Sitzung zum auswärtigen Mitgliede gewählte Herr Wladimir Demel in Wien macht brieflich darauf aufmerksam, dass statt seines Namens fälschlich der Name Deusel angefübrt worden sei.

Hr. Oppenheim bemerkt, dass dieser Febler die Folge eines unrichtig geschriebenen Vorschlagszettels sei und knüpft die wiederholte Aufforderung daran, die Namen der Vorgeschlagenen möglichst deutlich und correct und stets mit Vornamen anzugeben; beides sei für die Führung richtiger Listen anumgänglich nötbig.

Derselbe macht im Auftrage von Hrn. Henninger die Mittheilung, dass dieser Chemiker von einer Krankheit, welche seine Thätigkeit unterbrochen habe, genesen, binnen Kurzem seine Correspondenz aus Paris wieder anfnehmen werde.

Gewählt werden:

1) als einheimische Mitglieder:

die Herren:

Dr. Paul Wittelhöfer, Oranienburgerstrasse 5. II. N., Gustav Meyer, Assistenten am Anorganischen Emanuel Glatzel, Laborat. der K. Gewerbeakademie, Henry Liepmann, Universitätslaboratorium, Carl Reimer, stud. phil., Hafenplatz 3, SW., Leo Lewy, Rosenthalerstrasse 53. III. C., Ludwig Landshoff, stud. phil., Bellevuestrasse 12, w., James Morris, stud. phil., Marienstrasse 16, NW., Wilhelm Wydler, Assistent am pharmakologischen Institut der Universität, Louisenstrasse 35 ,

Dr. Gustav A. Scheeffer, Blumenstrasse 7 A.; 
2) als auswärtige Mitglieder:

die Herren:

P. Brouwerk, Lehrer der Chemie und Mineralogie an der höheren Bürgerschule in Deventer (Holland),

Dr. Lauts ch, Oberlebrer, Insterburg,

Spoerl, Chemiker in Weltingen, Poststation Schillingsfürst in Bayern,

Ach. Le Bel, Préparateur de Chimie, Faculté de Médicine, Paris,

Albert Ungerer, Simmering bei Wien,

Dr. Rudolf Messel, Director der Chemischen Fabrik Squire, Chapman \& Co., Silvertown, Victoria Docks E. London,

Dr. B. van der Meulen in Sappemeer, Provinz Groningen, Holland,

Dr. Polenskei, New-York, (?)

Dr. Rudolph Luboldt, Adr. Gehe \& Co., Dresden,

Dr. Ed. Chambon, Victoriastrasse 4 A., Deutz a. Rh.,

Dr. Christian Gänge, Physiologisches Laboratorium, Jena,

Dr. Heusinger von Waldegg in Wetzlar,

Dr. Eugen Schaal, Chemiker der Alizarinfabrik von Hru. Hufschmidt, Vogelsane, Elberfeld,

Dr. - Röderer, Assistent,

Dr. - Oppermann,

Dr. - Mählmann,

Dr. - Kerstein,

-.. Moser, stud. phil.,

- Bittmann, stud. phil.,

- Mittelmann, stud. phil.,

Universitätslaboratorium

Freiburg i. Br.,

Hermann Frank, Apotheker, Freiburg i. Br.,

Max Schmöger, Assistent an der landwirthschaftlichen

Versuchsstation in Möckern bei Leipzig,

Bruno Goës, Chemiker an der Fabrik der HH. Rindschedler und Busch in Basel,

Friedrich Egli, 23 Neumarkt, Zürich,

- Elb, Fabrikbesitzer in Blasewitz bei Dresden,

Dr. Arthur Forster, Assistent am Chemischen Laboratorium des Polytechnicums,

Dresden,

Alw in Hein rich Goldberg, Ritterstr. 4, II,

Ernst Louis, 1. III. Odeonplatz,

H. Bührig, 7. II. Gabelsberger Strasse, München, 
F. Clausnizer,

H. Körner,

S. Binswanger,

E. Seelig,

Otto Binder,

H. Kiliani,

V. Trautmann,

Caesar Rytel,

J. v. Hoermann,

J. M. Speucer,

A. Könyöky,

Fr. Fischer,

J. Ottmann,

K. Richter,

Th. Göring,

A. King,

\section{Chemisches Laboratorium}

des

Polytechnicum in München.

Für die Bibliothek sind eingegangen:

Als Geschenk:

Fehling: Neues Handwörterbuch der Chemie. Bd, II. Liefg. 6. Brauschweig 1876. (Vom Herausgeber.)

Dr. E. Czyrnianski: Teoryja mechaniozno-chemiczna. Kraków 1876. (Vom Verfasser.)

Alarignac: Sur les chaleurs spécifiques des solutions salines. Sep.-Abdr. (Yom Verfasser.)

13r. Kerl: Repertorium der technischen Literatur. Neue Folge, die Jahre 1869 bis 1874. umfassend. Leipzig 1875. (Vom Verf.)

i. W. Hofmann: The Life-Work of Liebig, in experimental and philosophic Chemistry; with allusions to his infuence on the development of the collateral sciences and of the useful arts. A Discourse, delivered to the Fellows of the Chemical Society of London, on March the 18th, 1875. London 1876. (Vom Verfasser.)

Als Austausch:

Justus Liebig's Annalen der Chemie. Ba, 180, Ieft 1 u. 2.

Chemisches Centralblatt. No. 7 u. 8 .

Deutsche Industriezcitung. No. 8.

Neues Repertorium für Pharmacie. Bd. XXV, Heft 1.

Verhandlungen der K. K. geologischen Reichsanstalt. No. 2.

Bulletin de la Bocieté ohimique de Paris. T. XXV, No. 4.

Bulletin de la Société industrielle de Rouen. $3^{\circ}$ année. No. 4. (Oct. à Dec. 1875.) lievue kebdomadaive de Chimie. No. 6, 7.

Revue scientifique. No. 34.

Journal der Russischen chemischen Gesellschaft. T. VIIT, No. 1.

\section{Durch Kauf:}

Dingler's polytechnisches Journal. Bd. 219 , Heft 4 u. 5 .

Comptes rendus. Tome LXXXII. (1876, I.) No. $1-7$. 